\title{
Applications of a Compact "Water Window" Source for Investigations of Nanostructures Using SXR Microscope
}

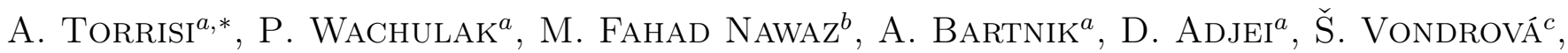 \\ J. TURŇOVÁ ${ }^{c}$, A. JANČAREK ${ }^{b}$ AND H. FiEdOROWICZ ${ }^{a}$ \\ ${ }^{a}$ Institute of Optoelectronics, Military University of Technology, S. Kaliskiego 2, 00-908 Warsaw, Poland \\ ${ }^{b}$ Faculty of Nuclear Sciences and Physical Engineering, Czech Technical University in Prague, \\ Brehova 7, 11519 Prague 1, Czech Republic \\ ${ }^{c}$ Faculty of Biomedical Engineering, Czech Technical University in Prague, \\ Brehova 7, 11519 Prague 1, Czech Republic
}

\begin{abstract}
A compact soft X-ray microscope based on a nitrogen double-stream gas puff target soft X-ray source, operating at He-like nitrogen spectral line at the wavelength of $\lambda=2.88 \mathrm{~nm}$ is presented. The desk-top size microscope was successfully demonstrated in transmission mode using the Fresnel zone-plate objective and it is suitable for soft X-ray source microscopy in the "water window" spectral range $(\lambda=2.3 \div 4.4 \mathrm{~nm})$. Details about the soft X-ray source source, the microscope and an example of application in the biomedical field are shown and discussed.
\end{abstract}

DOI: 10.12693/APhysPolA.129.169

PACS: 52.38.-r, 42.30.-d, 42.62.-b, 87.59.-e, 68.37.Yz, 07.85.Tt, 41.50.+h, 42.79.Dj

\section{Introduction}

Recent developments in the field of nanotechnology require the improvement of nanometer imaging systems. The introduction of compact sources of bright soft X-ray source (SXR) radiation permits the development of a table-top microscope that can magnify images of nanoscale objects highlighting detailed features approaching a $60 \mathrm{~nm}$ half-pitch spatial resolution, with short exposures and without the use of large facility such as synchrotrons.

In this work, we present a compact desk-top microscope, using a double stream gas puff target source with the Fresnel zone plate objective [1].

\section{Experimental setup}

Herein, we present a compact desk-top SXR microscope (Fig. 1) with a very simple construction, for high resolution imaging, employing nitrogen plasma SXR radiation. The radiation is produced by focusing an infrared Nd:YAG laser pulse, $\lambda=1064 \mathrm{~nm}$ (EKSPLA, pulse duration of $4 \mathrm{~ns}$ and pulse energy of $0.74 \mathrm{~J}$, at $1-10 \mathrm{~Hz}$ repetition rate) onto a double-stream gas puff target. The gaseous target is formed by two circular symmetric nozzles. The inner nozzle injects small amount of nitrogen, while the outer nozzle - a low-Z gas (helium), into the vacuum. The helium pressure reduces nitrogen density gradient along the nozzle axis, in order to obtain higher gas density further away from the nozzle, avoiding the nozzle ablation by repetitive plasma formation and improving the SXR emission yield. The pressures

*corresponding author; e-mail: alfio.torrisi@wat.edu.pl

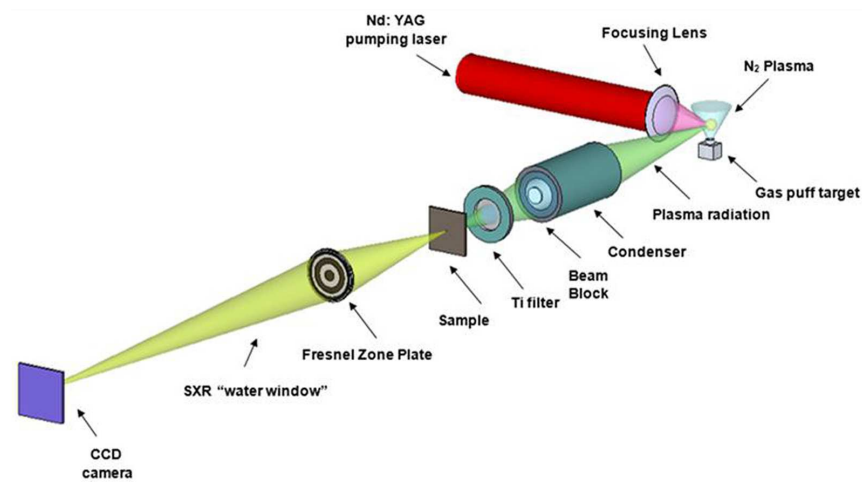

Fig. 1. Scheme of the "water window" SXR microscope based a double-stream nitrogen/helium gas puff target source, Fresnel zone plate objective and ellipsoidal condenser.

(6 bar for helium and 8 bar for nitrogen), were optimized to obtain high photon flux at the sample plane. More details about the SXR source may be found in [2].

The use of the gas puff target eliminates debris production and possible laser reflections, due to the under critical plasma density. Additionally, produced X-rays at large angles $\left(90^{\circ}\right)$ with respect to the incident laser beam drastically reduce the plasma ion emission in the direction of X-rays.

The SXR radiation from the plasma is focused by an ellipsoidal axi-symmetrical SXR condenser (Rigaku) coated with nickel that can efficiently focus the SXR radiation onto the object. A titanium filter (Lebow) allows to cut off $\mathrm{H}$-like line almost completely, so the main part of the energy is in a single line $(\lambda=2.88 \mathrm{~nm})$, in the "water window" spectral range [3-6].

The measured focal spot size is $1 \mathrm{~mm}$ in diameter, in the second focal plane of the condenser, where the sample 
is placed (140 mm downstream the condenser). Photon flux measurement was performed in the second focal plane of the condenser mirror using an AXUV 100 silicon $p-n$ junction photodiode (International Radiation Detectors, Inc.), with a Ti filter in place. The measured photon flux was $(7.9 \pm 0.2) \times 10^{9}$ photons/pulse, that corresponds to energy of $561 \pm 17 \mathrm{~nJ} /$ pulse at $\lambda=2.88 \mathrm{~nm}$ [7]. The spectrum from nitrogen plasma in the SXR region consists of two major lines: hydrogen-like nitrogen at $2.48 \mathrm{~nm}$ wavelength and helium-like nitrogen at $2.88 \mathrm{~nm}$. The later line was selected for imaging experiment by the use of $200 \mathrm{~nm}$ thick titanium filter.

A silicon nitride Fresnel zone plate (FZP) objective (diameter $250 \mu \mathrm{m}$ and outer zone width of $30 \mathrm{~nm}$, thickness $400 \mathrm{~nm}$ ) was used to magnify image of the sample onto a back-illuminated SXR-sensitive CCD camera (Andor iKon DO-934N, $1024 \times 1024$ pixels) in transmission mode. The numerical aperture of the zone plate, $\mathrm{NA}_{\text {ZP }}=0.048$, was matched with the numerical aperture of the condenser $\mathrm{NA}_{\mathrm{C} \text { out }}=0.05$, generating incoherent illumination [8]. This setup allowed a sub-60 nm spatial resolution SXR microscopy to be demonstrated [7] so far.

\section{Results and discussions}

As a demonstration, we show an SXR image of dehydrated CT 26 fibroblasts, derived from Mus musculus colon carcinoma (strain BALB/c), prepared on top of a $100 \mathrm{~nm}$ thick $\mathrm{Si}_{3} \mathrm{~N}_{4}$ membrane. Figure 2 shows direct comparison between images acquired by a visible light microscope (a, c, e, g) and SXR images of the cells $(b, d, f, h)$. The SXR images were acquired with 200 SXR pulses, 22 s exposure time, source repetition rate of $10 \mathrm{~Hz}$, detector temperature $-20^{\circ} \mathrm{C}$, while the visible light microscopy images were obtained with $40 \times$ objective and $\mathrm{NA}=0.7$.

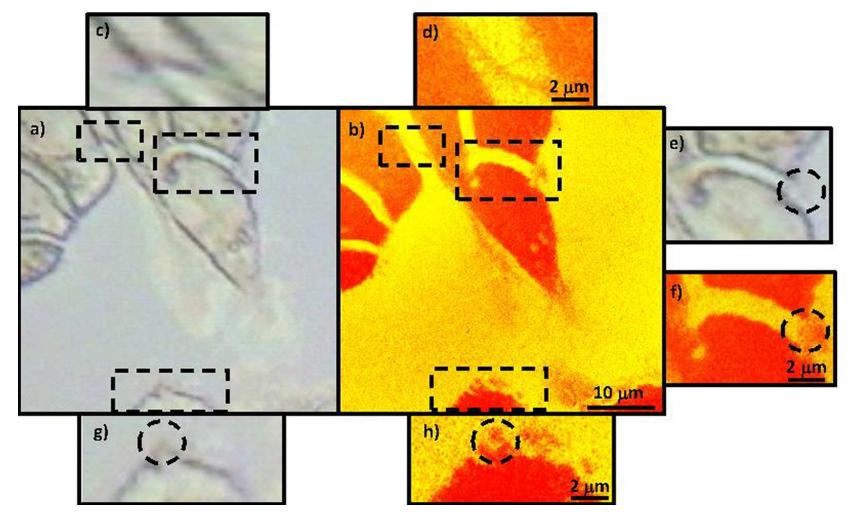

Fig. 2. Comparison between visible light microscopy images (a,c,e,g) and SXR images (b,d,f,h, false colors) of dehydrated CT 26 fibroblasts, derived from colon carcinoma Mus musculus (strain BALB/c). It is possible to observe cells with internal structure using visible light microscope (phase contrast mode) and due to the modulation in the transmittance of the SXR light through the sample in the SXR images.
The SXR images show enhanced contrast and spatial resolution due to shorter wavelength, beyond the capabilities of diffraction limited visible light microscopes. Due to a superior spatial resolution, achieved by this microscope, it is possible to resolve some internal structures within the cells with dimensions of the order of $\sim 200 \mathrm{~nm}$. Comparison between small sections from visible light images and SXR images, showing superior resolution and contrast, are shown in Fig. 2 with black dotted boxes. In Fig. $2 \mathrm{f}$ it is possible to observe how the high absorption coefficient at SXR wavelengths enhance the optical contrast; the SXR image is clearly sharper than the optical one in Fig. 2e and shows small features, observable due to the absorption contrast (encircled in Fig. $2 \mathrm{f}$ and e for comparison), which are not visible in Fig. 2e due to low phase contrast. Looking at Fig. 2h, it is possible also to see an improvement of the spatial resolution using SXR radiation; in fact, it is possible to resolve some small features (also encircled for clarity) that are not visible with the optical microscope (Fig. 2g).

\section{Conclusions}

We reported a desk-top, compact "water window" SXR microscope, based on a nitrogen double-stream gas puff target SXR source and Fresnel zone plate objective and applied it to study the real samples. The half-pitch spatial resolution achievable with such SXR microscope is $\approx 60 \mathrm{~nm}$, with exposure time as low as a few s. An example application, presented in this paper, shows applicability of such "water window" microscope to image biological samples with nanometer spatial resolution.

\section{Acknowledgments}

This work is supported by the National Centre for Science, award \#DEC-2011/03/D/ST2/00296 and National Centre for Research and Development, Lider programme, award \#LIDER/004/410/L-4/12/NCBR/2013. The authors acknowledge also financial support from the EU FP7 Erasmus Mundus Joint Doctorate Program EXTATIC under framework partnership agreement FPA2012-0033 and from the 7th Framework Programme's Laserlab Europe III project No. 284464.

\section{References}

[1] A.G. Michette, Optical Systems for Soft X-Rays, Plenum Press, New York 1986.

[2] P.W. Wachulak, A. Bartnik, M. Skorupka, J. Kostecki, R. Jarocki, M. Szczurek, L. Wegrzynski, T. Fok, H. Fiedorowicz, Appl. Phys. B 111, 239 (2013).

[3] K.W. Kim, Y. Kwon, K.Y. Nam, J.H. Lim, K.G. Kim, K.S. Chon, B.H. Kim, D.E. Kim, J.G. Kim, B.N. Ahn, H.J. Shin, S. Rah, K.H. Kim, J.S. Chae, D.G. Gweon, D.W. Kang, S.H. Kang, J.Y. Min, K.S. Choi, S.E. Yoon, E.A. Kim, Y. Namba, K.H. Yoon, Phys. Med. Biol. 51, N99 (2006). 
[4] L.B. Da Silva, J.E. Trebes, R. Balhorn, S. Mrowka, E. Anderson, D.T. Atwood, T.W. Barbee Jr., J. Brase, M. Corzett, J. Gray, J.A. Koch, C. Lee, D. Kern, R.A. London, B.J. MacGowan, D.L. Mathews, Science 258, 269 (1992).

[5] M. Berglund, L. Rymell, M. Peuker, T. Wilhein, H.M. Hertz, J. Microsc. 197, 268 (2000).

[6] M. Müller, T. Mey, J. Niemeyer, K. Mann, Opt. Express 22, 23489 (2014).
[7] P.W. Wachulak, A. Torrisi, A. Bartnik, D. Adjei, J. Kostecki, L. Wegrzynski, R. Jarocki, M. Szczurek, H. Fiedorowicz, Appl. Phys. B 118, 573 (2015).

[8] J.M. Heck, D.T. Attwood, W. Meyer-Ilse, E.H. Anderson, J. X-Ray Sci. Technol. 8, 95 (1998). 